\title{
Electron Beam-Induced Transformation in High-Density Amorphous Ices
}

\author{
Hongyi Xu, Jonas Ångström, Tobias Eklund, and Katrin Amann-Winkel* \\ Cite This: J. Phys. Chem. B 2020, 124, 9283-9288 \\ Read Online
}

ABSTRACT: Amorphous ice is commonly used as a noncrystalline matrix for protecting sensitive biological samples in cryogenic electron microscopy (cryo-EM). The amorphization process of water is complex, and at least two amorphous states of different densities are known to exist, high- and lowdensity amorphous ices (HDA and LDA). These forms are considered to be the counterparts of two distinct liquid states, namely, high- and low-density liquid water. Herein, we investigate the HDA to LDA transition using electron diffraction and cryo-EM. The observed phase transition is induced by the impact of electrons, and we discuss two different mechanisms, namely, local heating and beam-induced motion of water molecules. The temperature increase is estimated by comparison with X-ray scattering experiments on identically prepared samples. Our results suggest that HDA, under the conditions used in our cryo-EM measurements, is locally heated above its glass-transition temperature.

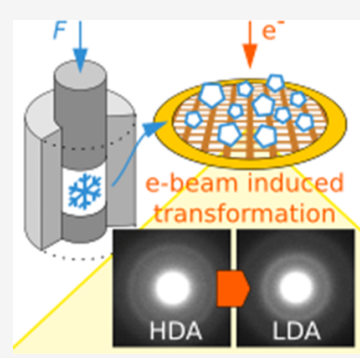

\section{INTRODUCTION}

Cryogenic electron microscopy (cryo-EM) is a modern and well-established method in structural biology. One of the most important breakthroughs of cryo-EM was the ability to vitrify liquid water. ${ }^{1,2}$ This can be achieved by jet-freezing, ${ }^{1}$ plungefreezing, ${ }^{2}$ or vitrification inside a vacuum chamber. ${ }^{3}$ Neutronand X-ray diffraction experiments investigated the structure of these hyperquenched glassy water (HGW) samples as well as the vapor-deposited ice (amorphous solid water, ASW), showing that both belong to the family of so-called lowdensity amorphous ice (LDA) and are almost identical. ${ }^{4}$ LDAs have a density ${ }^{5}$ of $0.94 \mathrm{~g} / \mathrm{cm}^{3}$ and appear as amorphous in neutron- and X-ray diffraction measurements with a first sharp diffraction peak (FSDP) around $Q=1.7 \AA^{-1}$. The amorphous ice formed on EM grids after plunge-freezing shows a smooth ring in the electron diffraction (ED) pattern at the same $Q$ position (given $d=2 \pi / Q=3.7 \AA$ ) ${ }^{6-8}$ According to the position of the FSDP, plunge-frozen vitrified water is identical to $\mathrm{LDA}^{9}$ and ASW. ${ }^{6}$ The recent pair distribution function calculations suggest small differences between plunge-frozen water and $\mathrm{LDA}^{\text {. }}$

A distinct denser form of amorphous ice can be prepared by compression of hexagonal ice at low temperatures. ${ }^{11}$ Highdensity amorphous ice (HDA) exists in various substates, ${ }^{5}$ with the two extreme cases being very high-density amorphous ice (VHDA) and expanded HDA (eHDA). ${ }^{12}$ The amorphous ices are suggested to undergo a glass-liquid transition to its corresponding liquid states of water, high- and low-density liquids (HDL and LDL, respectively). ${ }^{13-16}$ Previously, a calorimetric glass transition was detected in HDA at $116 \mathrm{~K}^{17}$ and in LDA at $136 \mathrm{~K}^{18}$ Some experimental studies support the finding of the glass-liquid transition, ${ }^{17-21}$ while others doubt the relationship of amorphous ice and liquid water. ${ }^{2-24}$ Computer simulations using different water models have suggested different thermodynamic scenarios over the last few decades. ${ }^{25-27}$ However, recent results show that different water models also converge to the same picture of two liquid states of water and the existence of a liquid-liquid critical point (LLCP). ${ }^{26,28}$ Experimentally, the solid-state HDA and LDA can be reversibly converted into each other by compressiondecompression. ${ }^{29,30}$ The temperature-induced transition at ambient pressure is irreversible; ${ }^{9,11,31} \mathrm{X}$-ray diffraction studies showed that eHDA transforms to HDL and finally to LDL in a diffusive manner at around $140 \mathrm{~K},{ }^{21}$ being consistent with the LLCP model. ${ }^{26,28}$ Other experimental studies also report on an HDA-like state formed by vapor deposition at $10 \mathrm{~K}^{32}$ and by irradiation of plunge-frozen amorphous ice with an electron beam using transmission electron microscopy (TEM) at $T<$ $30 \mathrm{~K} .{ }^{8,33}$ This ice is slightly less compact compared to HDA formed at high pressures.

Electron beam-induced movement of vitrified specimens and translational motion of single particles within the amorphous ice matrix or even the motion of the ice itself has been discussed widely in the EM literature. ${ }^{7,33-35}$ The random motion of particles embedded in vitrified water is one of the challenges in cryo-EM, ${ }^{35}$ as it leads to blurring of the image and reduces the contrast, in particular, for small objects. ${ }^{7} \mathrm{~A}$ major source of motion has been connected to radiolysis and the electrostatic charging of the amorphous ice and the carbon support film on the grid itself. ${ }^{7,8}$ Some additional motion,

Received: September 9, 2020

Revised: September 15, 2020

Published: September 30, 2020 
however, is related to beam-induced changes in the ice layer. ${ }^{34,35}$ Understanding the origin of this motion is important to find possible ways to avoid this effect and to learn about the nature of vitrified water itself.

\section{METHODS}

While amorphous ice in EM studies is typically made by plunge-freezing, the present study instead focuses on ED of the high-pressure forms eHDA and VHDA as starting materials. The amorphous ice samples are made ex situ by pressureinduced amorphization using a piston cylinder setup, as described earlier; ${ }^{5,11}$ details can be found in the Supporting Information. The amorphous ice samples are ground to a fine powder inside a bath of liquid nitrogen. A few fragments of the crushed ice are collected by dipping a TEM grid (QUANTIFOIL R2/1, Cu, 300 mesh) into the ice powder. Using this procedure gives a unique opportunity to handle samples that cannot be mounted onto an EM grid in the conventional way. The grid was transferred onto a cryotransfer tomography holder (Gatan type-914) and inserted into a JEOL 2010 TEM with the $\mathrm{LaB}_{6}$ filament. ED experiments were performed under a parallel electron illumination with an acceleration voltage of $200 \mathrm{kV}$. We used an electron beam of $6 \mu \mathrm{m}$ in diameter with an estimated dose rate of $0.1 \mathrm{e}^{-} \mathrm{s}^{-1} \AA^{-2}$. ED patterns were collected continuously using an ultrafast hybrid pixel detector (Timepix, Amsterdam Scientific Instruments, $8 \mathrm{~ms}$ dead time, $512 \times 512$ pixels $)$. TEM images were collected using a side-entry Gatan Orius SC200 detector.

\section{RESULTS}

Figure 1 (upper row) shows the TEM bright-field images of amorphous ice cold-loaded under liquid nitrogen on a cryotransfer holder. Our sample preparation method does not provide us with a homogenous ice film but rather with many fragments of amorphous ice on top of a $\mathrm{Cu}$ grid. This way small crystalline ice flakes also get onto the grid during the loading procedure, as visible by the hexagonal shape of the
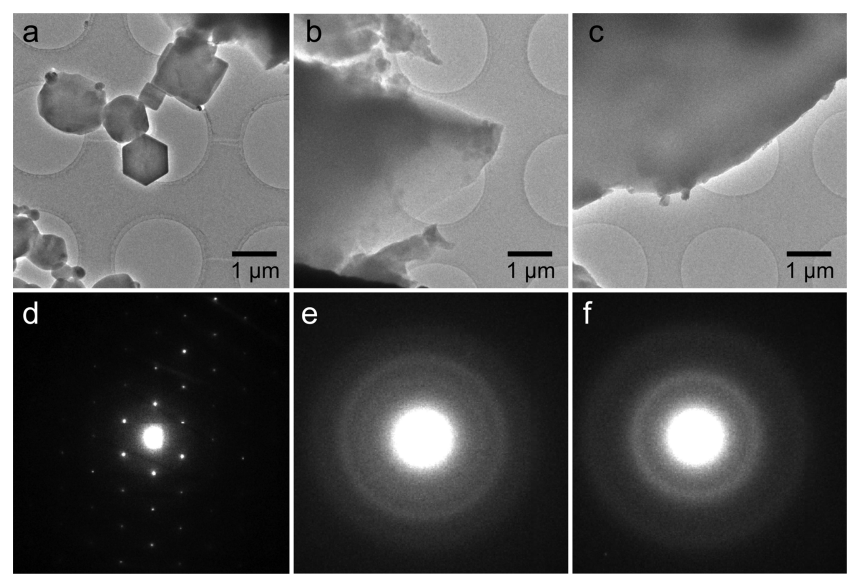

Figure 1. TEM bright-field images with high magnification (upper row) and diffraction patterns (lower row) of hexagonal ice (a, d) and the amorphous ices VHDA (b, e) and LDA (c, f), formed after transition. Images are taken as representative examples. The ice samples were prepared following a well-established protocol and afterward ground to a fine powder inside a bath of liquid nitrogen. A few fragments of the crushed ice were collected by dipping a TEM grid (QUANTIFOIL R2/1, Cu, 300 mesh) in the ice powder. centered flake in Figure 1a. The corresponding diffraction pattern (bottom row, 1d) shows Bragg reflections of hexagonal ice. Amorphous ice fragments are instead larger without sharp edges (Figure 1b,c). We estimate the flakes to be on the order of 50-500 nm thickness, while they appear black (nontransparent) in the case of a larger thickness. Diffraction patterns taken from thin flakes show the characteristic smooth rings for amorphous ice. Figure le shows a VHDA sample, which can be transformed into LDA (1f), as seen by the change in the inner diffraction ring, and discussed in detail below.

Figure 2 shows diffraction measurements of a VHDA sample taken at around $98 \mathrm{~K}$. In total, 199 diffraction patterns were
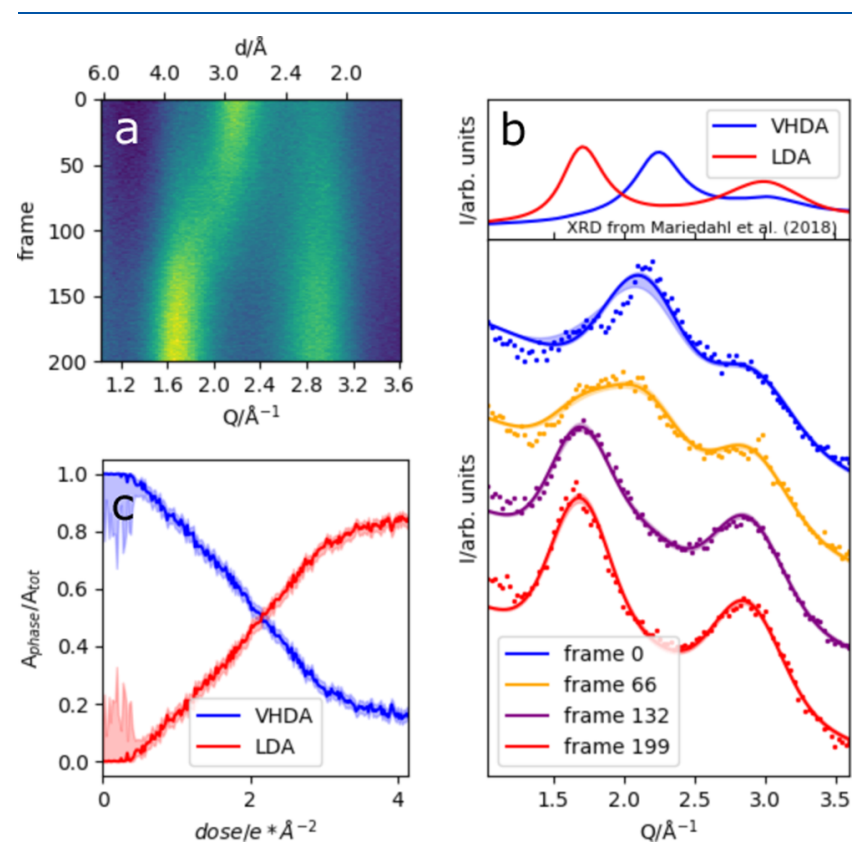

Figure 2. ED, the dose rate is $0.1 \mathrm{e}^{-} \mathrm{s}^{-1} \AA^{-2}$. The VHDA sample is measured at $98 \mathrm{~K}$; a series of 199 images are obtained with an exposure time of $0.2 \mathrm{~s} /$ frame. The angular integrated data (a) are fitted (b, bottom panel) and further analyzed by integration of the FSDP (c). Top panel in (b) shows X-ray diffraction data, reproduced from refs 21 and 36.

taken at the same spot (size of the selected area aperture: $2 \mu \mathrm{m}$ in diameter) using an exposure time of $0.2 \mathrm{~s} /$ pattern. Figure $2 \mathrm{a}$ shows the result obtained by integrating the diffuse diffraction rings (Figure 1e,f), a beam-induced transformation that takes place over time. Figure $2 b$ (lower panel) shows the integrated intensity for four selected time frames including a fit obtained by Bayesian inference (Supporting Information). For comparison, $I(Q)$ measured by X-ray scattering ${ }^{36}$ is plotted on top of Figure $2 \mathrm{~b}$. The first ED diffraction pattern (frame 0, blue) shows two rings positioned at distances $d_{1}=2.95 \AA$ and $d_{2}=$ $2.17 \AA$. The last diffraction pattern (frame 199, red) shows the first ring to be shifted to $d_{1}=3.73 \AA$. The position of the latter is consistent with the other ED patterns in the literature taken from plunge-frozen amorphous ice, ${ }^{9}$ thus the sample transformed to LDA. When converted to momentum transfer $Q$, the position of the FSDP is $Q_{1}^{\mathrm{VHDA}}=2.13 \AA^{-1}$ and $Q_{1}^{\mathrm{LDA}}=1.69$ $\AA^{-1}$. This value obtained for LDA is consistent with the X-ray scattering data. ${ }^{36}$ The position of the VHDA maximum instead differs from the literature. From X-ray scattering, we would expect the $Q$-position in VHDA to be around $Q_{1}^{\mathrm{VHDA}}=2.3 \AA^{-1}$. 
The sample-transfer process into the electron microscope, however, is much more difficult, and we cannot exclude partial transformation during the sample mounting. Calculating the ratio $A_{\text {phase }} / A_{\text {tot }}$ of the area under the peak of one phase, we can monitor the phase transformation (Figure $2 c$ ).

Considering the exposure time, acquisition time, and dead time of the detector, the transition is found to take place at around $20 \mathrm{~s}$. After the transformation to LDA, we continued the measurement for another $70 \mathrm{~s}$ (not shown here) and could not detect any further changes or any sign for crystallization. Figure 3 shows ED measurements of an eHDA sample. In
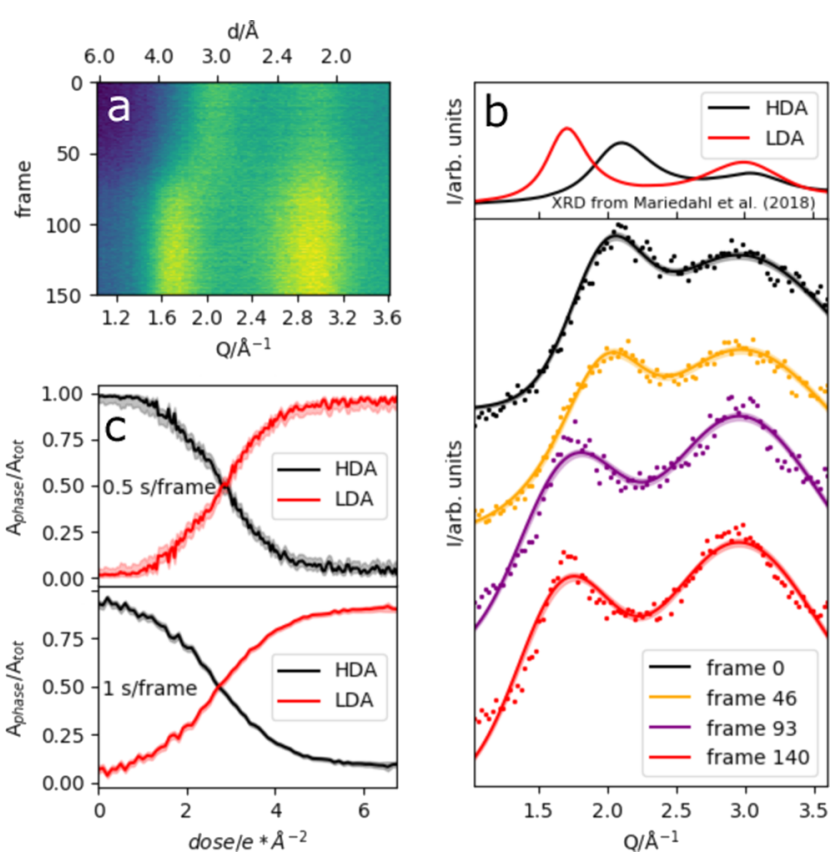

Figure 3. ED, the dose rate is $0.1 \mathrm{e}^{-} \mathrm{s}^{-1} \AA^{-2}$. The eHDA sample is measured at $98 \mathrm{~K}$; a series of 140 images are obtained with an exposure time of $0.5 \mathrm{~s} /$ frame. The angular integrated data (a) are fitted (b, bottom panel) and further analyzed by integration of the FSDP (c). (c-bottom) Additional measurement with 62 images with an exposure of $1 \mathrm{~s} /$ frame. Top panel in (b) shows X-ray diffraction data, reproduced from refs 21 and 36 .

total, 140 diffraction patterns were taken at the same spot, with an exposure time of $0.5 \mathrm{~s}$. The angular integrated data are shown in Figure 3a,b, and similar to the VHDA sample show a beam-induced transformation from eHDA to LDA. The first diffraction pattern (frame 0 , black curve) shows two rings positioned at distances $d_{1}=3.16 \AA$ and $d_{2}=2.11 \AA$, corresponding to momentum transfers of $Q_{1}^{\mathrm{eHDA}}=1.99 \AA^{-1}$ and $Q_{2}^{\text {eHDA }}=2.97 \AA^{-1}$. The last diffraction pattern (frame 140, red curve) shows the first ring at $d_{1}=3.77 \AA\left(Q_{1}^{\mathrm{LDA}}=1.67\right.$ $\left.\AA^{-1}\right)$. The obtained values are consistent with $\mathrm{X}$-ray ${ }^{36}$ and neutron diffraction studies. ${ }^{12}$ Figure $3 \mathrm{c}$ shows the transformation taking place after being exposed to $3 \mathrm{e}^{-} / \AA^{2}$, hence $\sim 60$ images were then taken with an exposure time of $0.5 \mathrm{~s}$. In a second, independent measurement (Figure $3 \mathrm{c}$ bottom), we choose an exposure time of $1 \mathrm{~s}$ and observed the transition after 30 images instead. The ED results are summarized in Table 1. Parameters derived from ED are obtained using a Bayesian model to fit $I(Q)$ (see the Supporting Information).

Additionally, we tested if the beam-induced transformation is locally confined by taking a series of diffraction patterns at two different spots, separated by $6 \mu \mathrm{m}$, using a selective area
Table 1. Values for the First and Second Diffraction Rings of Amorphous Ices, As Measured by ED and X-Ray Diffraction (Ref 36) of Similarly Made Samples ${ }^{a}$

\begin{tabular}{lcccccc}
\multicolumn{1}{c}{ sample } & $\begin{array}{c}d_{1} \\
(\AA)\end{array}$ & $\begin{array}{c}Q_{1}=2 \pi / d \\
\left(\AA^{-1}\right)\end{array}$ & $\begin{array}{c}Q_{1} \\
(\mathrm{X}-\text {-ray }) \\
\left(\AA^{-1}\right)^{36}\end{array}$ & $\begin{array}{c}d_{2} \\
(\AA)\end{array}$ & $\begin{array}{c}Q_{2} \\
\left(\AA^{-1}\right)\end{array}$ & $\begin{array}{c}(\mathrm{X}-\text { ray }) \\
\left(\AA^{-1}\right)^{36}\end{array}$ \\
VHDA & 2.95 & 2.13 & 2.30 & 2.17 & 2.90 & 3.05 \\
eHDA & 3.16 & 1.99 & 2.10 & 2.11 & 2.97 & 3.05 \\
LDA $^{\text {VHDA }}$ & 3.73 & 1.69 & 1.70 & 2.19 & 2.87 & 3.05 \\
LDA $^{\text {eHDA }}$ & 3.77 & 1.67 & 1.70 & 2.14 & 2.93 & 3.05 \\
HGW $_{\text {EM-lit (9) }}$ & 3.76 & & & 2.18 & &
\end{tabular}

${ }^{a}$ Because of experimental errors and geometrical reasons (see the Supporting Information), the values have an error in $Q$ of $\pm 0.04 \AA^{-1}$ around $Q_{1}$ and $\pm 0.08 \AA^{-1}$ around $Q_{2}$. The $\mathrm{X}$-ray values instead have a much smaller error $\left(<0.01 \AA^{-1}\right)$.

aperture size of $2 \mu \mathrm{m}$ in diameter (Figure $4 \mathrm{a}$, red circles). After monitoring the HDA to LDA transition in the first spot, we
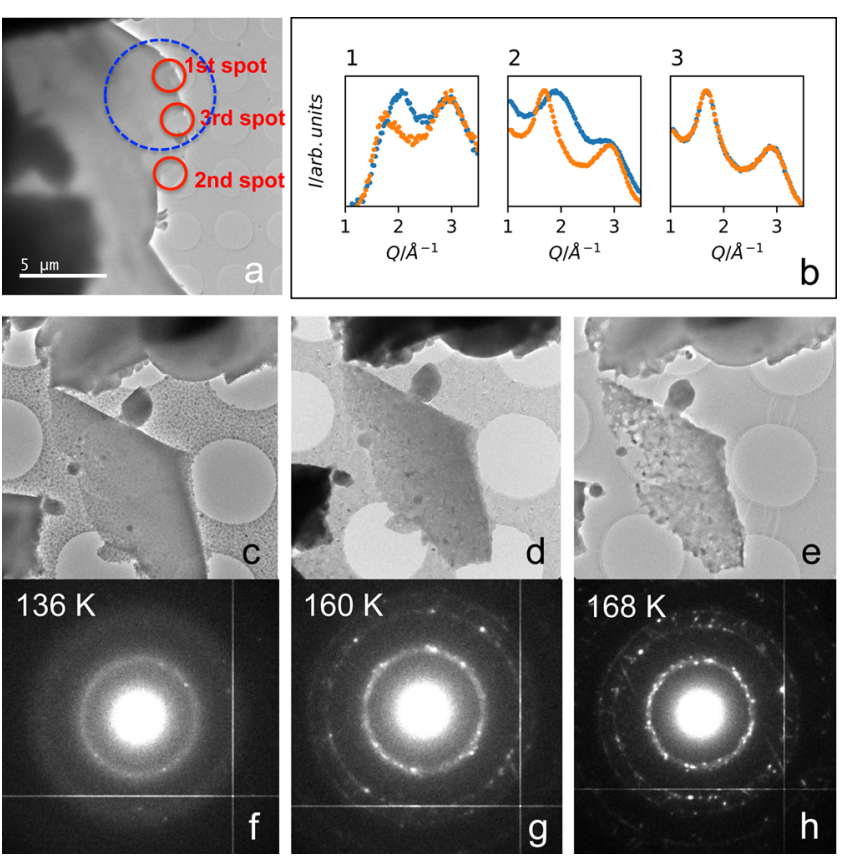

Figure 4. TEM bright-field images and the corresponding ED data. (a) Three different locations investigated on an eHDA sample using a selective area aperture size of $2 \mu \mathrm{m}$ (red circles). The blue dashed circle indicates the full area of illumination $(6 \mu \mathrm{m})$ while measuring spot 1. (b) Diffraction pattern of initial (blue) and final (orange) states at the three spots. Images $(c-h)$ show active heating of the sample holder during a separated experiment. The actual temperature at the tip of the holder is unknown, thus an offset to the given temperature cannot be excluded.

could identify HDA as the initial state in the second spot and monitored the transition in these locations, as described above. Figure $4 \mathrm{~b}$ shows the corresponding initial (blue) and final (orange) states. This indicates that the thermal conductivity of eHDA is small enough to confine the beam-induced transformation within the illuminated area. As reference, a third spot was taken within the previously irradiated area (big blue circle), confirming that the LDA formation had already taken place during the first illumination. In order to investigate crystallization of the sample, we raised the base temperature of the sample holder further (Figure $4 \mathrm{c}-\mathrm{h}$ ). At $136 \mathrm{~K}$, small nanometer-sized crystallites appear in the image as well as few 
Bragg peaks in the diffraction pattern (Figure 4c,f) on top of the amorphous ice. The small crystallites are visible both on the carbon-coated grid, at the edges of larger ice chunks, and within the almost transparent piece of ice, located in the center of Figure 4c. It seems that these nanocrystallites form within or on top of the amorphous ice. We assume that a very thin additional layer of water was formed during the sample transfer by vapor deposition of water that started to crystallize at 136 K. Crystallization kinetics of thin amorphous ice films ${ }^{37}$ and thickness-dependent growth rates are discussed in the literature. ${ }^{38}$ Here, the small crystallites grow in size when the base temperature of the sample holder is further raised to 160 $\mathrm{K}$ (Figure 4d). At this temperature, the thicker amorphous ice fragments also start to crystallize, as seen in both the image (4d) and the corresponding diffraction pattern ( $4 \mathrm{~g})$. The ice starts disappearing at a base temperature of $168 \mathrm{~K}$ due to partial evaporation, visible by a reduced size of the thin ice chunk (Figure 4e). Ice formation on top of the carbon coating has been reported earlier as so-called "leopard skin ice". ${ }^{39}$

\section{DISCUSSION}

In the present study, we investigate the high- to low-density transition starting from VHDA and eHDA using a cryo-EM setup. We observe a beam-induced rapid structural transformation to LDA after an accumulated dose of $2-3 \mathrm{e}^{-} / \AA^{2}$, while the sample holder was kept at $98 \mathrm{~K}$. This phase transition is clearly driven by the electron interaction and can be explained by a combination of different effects of radiation damage. ${ }^{40}$ Amorphous ices are metastable states, and the transition at 1 bar is driven by the difference in Gibbs free energy. In most experimental studies on amorphous ice, the phase transition is induced by active heating. ${ }^{31,41}$ In the following, we will discuss two possible mechanisms: (1) beaminduced local heating due to inelastic scattering and (2) beaminduced transition due to bond reorientation by direct electron interaction.

Calorimetry, ${ }^{31} \mathrm{X}_{\text {-ray, }}{ }^{41}$ and neutron scattering ${ }^{12}$ show that eHDA transforms upon heating above $127 \mathrm{~K}^{41}$ VHDA is thermally less stable than $\mathrm{eHDA}^{31}$ and transforms around 117 $\mathrm{K}$. This is reflected in our ED results, as VHDA starts to transform at a lower dose compared to eHDA. A primary ED study, using active heating of the sample holder, was reported earlier using unannealed HDA (uHDA) as the starting material, ${ }^{9}$ which is today known to be thermally distinctly less stable compared to VHDA and eHDA. ${ }^{31}$ The reported value $^{9}$ for the first diffraction ring in UHDA is observed at a distance $d_{1}=3.65 \AA$, which is located more toward the position of LDA and not consistent with X-ray data. Assuming the phase transition observed in our current study is driven by local heating, the heating would be caused by inelastic scattering that leads to plasmon and phonon excitation. Comparing the current ED results with the literature, we estimate a maximal increase in temperature to around $117 \mathrm{~K}$ after an accumulated dose of $2 \mathrm{e}^{-} / \AA^{2}$ in the case of VHDA and to at least $127 \mathrm{~K}$ at $3 \mathrm{e}^{-} / \AA^{2}$ for eHDA. This would correspond to a temperature increase of $\Delta T=37 \mathrm{~K}$, although the comparison can only give a rough estimate for the temperature rise. The exothermic transition continues to the low-density state. ${ }^{17} \mathrm{We}$ did not observe crystallization in our ED measurements up to an accumulated dose of $7 \mathrm{e}^{-} / \AA^{2}$. A general saturation of beam-induced effects at increasing dose rates is also discussed in the literature. ${ }^{42}$ Egerton et al. ${ }^{43}$ calculated the electron beam-induced heating effect on carbon for low-current densities from the inelastic scattering cross section, showing a temperature rise of $<1.5 \mathrm{~K}$. Heat simulations ${ }^{40}$ predicted that heating amorphous ice (LDA) by the electron beam only takes place at "very large dose rates (50 $\left.\mathrm{e}^{-} / \AA^{2} \mathrm{~s}\right)$ combined with a poor thermal contact between the grid and the cryo-holder" ${ }^{40}$ For medium dose rates, the simulations predict a temperature increase of only $3 \mathrm{~K}$ for a flux of $5 \mathrm{e}^{-} / \AA^{2} \mathrm{~s}$, however, assuming a thermal conductivity of $1.1 \mathrm{~W} / \mathrm{m} \mathrm{K}$ (LDA). The thermal conductivity $\kappa$ for bulk HDA is $\sim 0.6 \mathrm{~W} / \mathrm{m} \mathrm{K}$ and is demonstrated to be significantly lower compared to LDA or hexagonal ice, ${ }^{44}$ as well as to carbon. ${ }^{43}$ Possible grain boundaries within the ice flakes could lead to further decrease of the thermal conductivity, a phenomenon also observed in other materials. For example, the thermal conductivity for porous ASW was determined to be several orders of magnitude lower compared to LDA. ${ }^{44,45}$ The low thermal conductivity of HDA combined with the exothermic character of the phase transition, as well as potentially low thermal contact of the ice flakes to the grid and/or the grid to the sample holder (see the Supporting Information), could give rise to a sufficient temperature increase in our experiments. A temperature increase above $115 \mathrm{~K}$ will lead to a glass transition in $\mathrm{HDA}^{17,21}$

A second possible scenario describes the observed transition to be induced directly by bond-reorientation through electron interaction or radiolysis. The motion of water molecules in vitrified amorphous ice has been reported in other EM studies. $^{7,34,35,43}$ Some measurements ${ }^{7}$ showed that water molecules move $\sim 1 \AA$ [root mean square (rms) distance] for each $\mathrm{e}^{-} / \AA^{2}$, leading to a pseudo-Brownian motion of embedded particles. The same mechanism that leads to this motion of water molecules may also drive the metastable HDA into the more stable state LDA. LDA is a nonequilibrium state itself, and one might assume that the process continues until the equilibrium crystalline state is reached. The lack of crystallization after doubling the dose indicates the limitation of this process at the given flux. Active heating of the stage as shown in Figure 4 leads to direct crystallization of the whole sample. How does the observed motion ${ }^{7}$ compare to the diffusive behavior of ultraviscous water? Measurements using $\mathrm{X}$-ray photon correlation spectroscopy recently revealed that the observed transition happens in a diffusive manner by determining the diffusion coefficients during the eHDAHDL-LDL transition. ${ }^{21}$ This study ${ }^{21}$ determined the diffusion coefficient for HDL at $115 \mathrm{~K}$ to be $D=0.8 \mathrm{~nm}^{2} / \mathrm{s}$, which leads to a rms distance of $\sim 2 \mathrm{~nm} / \mathrm{s}$. Assuming a dose rate of $1 \mathrm{e}^{-} / \AA^{2}$ $\mathrm{s}$, there is a discrepancy of around 1 order of magnitude between the thermally induced motion and the beam-induced Brownian motion.

To summarize, under the conditions used in this cryo-EM study, the observed high- to low-density transition can be explained by two mechanisms: (1) local heating due to low thermal conductivity of HDA, the exothermic character of the observed transition as well as limitations of the new sample preparation method and (2) pseudo-Brownian motion of water molecules due to bond reorientation induced by the electron beam. We conclude that the combination of both processes causes the sample to undergo a first-order like phase transition through a glass transition. 


\section{ASSOCIATED CONTENT}

\section{(3) Supporting Information}

The Supporting Information is available free of charge at https://pubs.acs.org/doi/10.1021/acs.jpcb.0c08232.

Experimental details, data analysis, results and discussion of ED and X-ray scattering measurements, and additional references (PDF)

\section{AUTHOR INFORMATION}

\section{Corresponding Author}

Katrin Amann-Winkel - Department of Physics, Chemical Physics Division, AlbaNova University Center, Stockholm University, SE-10691 Stockholm, Sweden; 이이.org/00000002-7319-7807; Email: Katrin.amannwinkel@fysik.su.se

\section{Authors}

Hongyi Xu - Department of Materials and Environmental Chemistry, Stockholm University, SE-10691 Stockholm, Sweden; ㅇo이.org/0000-0002-8271-3906

Jonas Ångström - Department of Chemistry-Ångström Laboratory, Uppsala University, SE-75121 Uppsala, Sweden

Tobias Eklund - Department of Physics, Chemical Physics Division, AlbaNova University Center, Stockholm University, SE-10691 Stockholm, Sweden

Complete contact information is available at:

https://pubs.acs.org/10.1021/acs.jpcb.0c08232

\section{Notes}

The authors declare no competing financial interest.

\section{ACKNOWLEDGMENTS}

K.A.-W. acknowledges financial support from the Ragnar Söderbergs Stiftelse (Sweden) and European Research Council Advanced Grant WATER under Project (667205). H.X. acknowledges financial support from the Swedish Research Council (2017-05333). J.Å. acknowledges support from STandUP for Energy. We thank X.D. Zou, C.W Tai, A. Nilsson, F. Perakis, and P. Amann for helpful discussions about the manuscript.

\section{REFERENCES}

(1) Brüggeller, P.; Mayer, E. Complete vitrification in pure liquid water and dilute aqueous solutions. Nature 1980, 288, 569-571.

(2) Dubochet, J.; McDowall, A. W. Vitrification of Pure Water for Electron Microscopy. J. Microsc. 1981, 124, 3-4.

(3) Kohl, I.; Bachmann, L.; Hallbrucker, A.; Mayer, E.; Loerting, T. Liquid-Like Relaxation in Hyperquenched Water at $\leq 140 \mathrm{~K}$. Phys. Chem. Chem. Phys. 2005, 7, 3210-3220.

(4) Bowron, D. T.; Finney, J. L.; Hallbrucker, A.; Kohl, I.; Loerting, T.; Mayer, E.; Soper, A. K. The Local and Intermediate Range Structures of the Five Amorphous Ices at $80 \mathrm{~K}$ and Ambient Pressure: A Faber-Ziman and Bhatia-Thornton Analysis. J. Chem. Phys. 2006, 125, 194502.

(5) Loerting, T.; Winkel, K.; Seidl, M.; Bauer, M.; Mitterdorfer, C.; Handle, P. H.; Salzmann, C. G.; Mayer, E.; Finney, J. L.; Bowron, D. T. How Many Amorphous Ices Are There? Phys. Chem. Chem. Phys. 2011, 13, 8783-8794.

(6) Dubochet, J.; Lepault, J. CRYO-electron microscopy of vitrified water. J. Phys. Colloq. 1984, 45, C7-85-C7-94.

(7) McMullan, G.; Vinothkumar, K. R.; Henderson, R. Thon rings from amorphous ice and implications of beam-induced Brownian motion in single particle electron cryo-microscopy. Ultramicroscopy 2015, 158, 26-32.
(8) Heide, H.-G.; Zeitler, E. The physical behavior of solid water at low temperatures and the embedding of electron microscopical specimens. Ultramicroscopy 1985, 16, 151-160.

(9) AL-Amoudi, A.; Dubochet, J.; Studer, D. Amorphous solid water produced by cryosectioning of crystalline ice at $113 \mathrm{~K}$. J. Microsc. 2002, 207, 146-153.

(10) Souza Junior, J. B.; Schleder, G. R.; Colombari, F. M.; de Farias, M. A.; Bettini, J.; van Heel, M.; Portugal, R. V.; Fazzio, A.; Leite, E. R. Pair Distribution Function from Electron Diffraction in Cryogenic Electron Microscopy: Revealing Glassy Water Structure. J. Phys. Chem. Lett. 2020, 11, 1564-1569.

(11) Mishima, O.; Calvert, L. D.; Whalley, E. "Melting ice" I at $77 \mathrm{~K}$ and $10 \mathrm{kbar}$ : a new method of making amorphous solids. Nature 1984, 310, 393-395.

(12) Nelmes, R. J.; Loveday, J. S.; Strässle, T.; Bull, C. L.; Guthrie, M.; Hamel, G.; Klotz, S. Annealed High-Density Amorphous Ice Under Pressure. Nat. Phys. 2006, 2, 414-418.

(13) Mishima, O.; Stanley, H. E. The relationship between liquid, supercooled and glassy water. Nature 1998, 396, 329-335.

(14) Debenedetti, P. G.; Stillinger, F. H. Supercooled liquids and the glass transition. Nature 2001, 410, 259-267.

(15) Amann-Winkel, K.; Böhmer, R.; Fujara, F.; Gainaru, C.; Geil, B.; Loerting, T. Colloquium: Water's Controversial Glass Transitions. Rev. Mod. Phys. 2016, 88, 011002.

(16) Handle, P. H.; Loerting, T.; Sciortino, F. Supercooled and glassy water: Metastable liquid(s), amorphous solid(s), and a noman's land. Proc. Natl. Acad. Sci. U.S.A. 2017, 114, 13336-13344.

(17) Amann-Winkel, K.; Gainaru, C.; Handle, P. H.; Seidl, M.; Nelson, H.; Bohmer, R.; Loerting, T. Water's Second Glass Transition. Proc. Natl. Acad. Sci. U.S.A. 2013, 110, 17720-17725.

(18) Johari, G. P.; Hallbrucker, A.; Mayer, E. The Glass-Liquid Transition of Hyperquenched Water. Nature 1987, 330, 552-553.

(19) Smith, R. S.; Kay, B. D. The existence of supercooled liquid water at 150 ?K. Nature $1999,398,788-791$.

(20) Andersson, O. Glass-Liquid Transition of Water at High PressureGlass-liquid transition of water at high pressure. Proc. Natl. Acad. Sci. U.S.A. 2011, 108, 11013-11016.

(21) Perakis, F.; Amann-Winkel, K.; Lehmkühler, F.; Sprung, M.; Mariedahl, D.; Sellberg, J. A.; Pathak, H.; Späh, A.; Cavalca, F.; Schlesinger, D.; et al. Diffusive dynamics during the high-to-low density transition in amorphous ice. Proc. Natl. Acad. Sci. U.S.A. 2017, $114,8193-8198$.

(22) Tse, J. S.; Klug, D. D. Pressure Amorphized Ices - An Atomistic Perspective. Phys. Chem. Chem. Phys. 2012, 14, 8255-8263.

(23) Shephard, J. J.; Ling, S.; Sosso, G. C.; Michaelides, A.; Slater, B.; Salzmann, C. G. Is High-Density Amorphous Ice Simply a "Derailed" State along the Ice I to Ice IV Pathway? J. Phys. Chem. Lett. 2017, 8, 1645-1650.

(24) Tulk, C. A.; Molaison, J. J.; Makhluf, A. R.; Manning, C. E.; Klug, D. D. Absence of amorphous forms when ice is compressed at low temperature. Nature 2019, 569, 542-545.

(25) Gallo, P.; Amann-Winkel, K.; Angell, C. A.; Anisimov, M. A.; Caupin, F.; Chakravarty, C.; Lascaris, E.; Loerting, T.; Panagiotopoulos, A. Z.; Russo, J.; Sellberg, J. A.; Stanley, H. E.; Tanaka, H.; Vega, C.; Xu, L.; Pettersson, L. G. M. Water: A tale of two liquids. Chem. Rev. 2016, 116, 7463-7500.

(26) Poole, P. H.; Sciortino, F.; Essmann, U.; Stanley, H. E. PhaseBehavior of Metastable Water. Nature 1992, 360, 324-328.

(27) Palmer, J. C.; Martelli, F.; Liu, Y.; Car, R.; Panagiotopoulos, A. Z.; Debenedetti, P. G. Metastable Liquid-Liquid Transition in a Molecular Model of Water. Nature 2014, 510, 385-388.

(28) Debenedetti, P. G.; Sciortino, F.; Zerze, G. H. Second critical point in two realistic models of water. Science 2020, 369, 289-292.

(29) Mishima, O.; Calvert, L. D.; Whalley, E. An Apparently FirstOrder Transition Between Two Amorphous Phases of Ice Induced by Pressure. Nature 1985, 314, 76-78.

(30) Shen, G.; Smith, J. S.; Kenney-Benson, C. Nature of polyamorphic transformations in $\mathrm{H} 2 \mathrm{O}$ under isothermal compression and decompression. Phys. Rev. Mater. 2019, 3, 073404. 
(31) Winkel, K.; Mayer, E.; Loerting, T. Equilibrated High-Density Amorphous Ice and its First-Order Transition to the Low-Density form. J. Phys. Chem. B 2011, 115, 14141-14148.

(32) Venkatesh, C. G.; Rice, S. A.; Narten, A. H. Amorphous Solid Water: An X-ray Diffraction Study. Science 1974, 186, 927.

(33) Wright, E. R.; Iancu, C. V.; Tivol, W. F.; Jensen, G. J. Observations on the behavior of vitreous ice at $\sim 82$ and $\sim 12 \mathrm{~K}$. J. Struct. Biol. 2006, 153, 241-252.

(34) Brilot, A. F.; Chen, J. Z.; Cheng, A.; Pan, J.; Harrison, S. C.; Potter, C. S.; Carragher, B.; Henderson, R.; Grigorieff, N. Beaminduced motion of vitrified specimen on holey carbon film. J. Struct. Biol. 2012, 177, 630-637.

(35) Henderson, R.; McMullan, G. Problems in obtaining perfect images by single-particle electron cryomicroscopy of biological structures in amorphous ice. Microscopy 2013, 62, 43-50.

(36) Mariedahl, D.; Perakis, F.; Späh, A.; Pathak, H.; Kim, K. H.; Camisasca, G.; Schlesinger, D.; Benmore, C.; Pettersson, L. G. M.; Nilsson, A.; et al. X-ray Scattering and O-O Pair-Distribution Functions of Amorphous Ices. J. Phys. Chem. B 2018, 122, 76167624.

(37) Smith, R. S.; Matthiesen, J.; Knox, J.; Kay, B. D. Crystallization Kinetics and Excess Free Energy of $\mathrm{H} 2 \mathrm{O}$ and D2O Nanoscale Films of Amorphous Solid Water. J. Phys. Chem. A 2011, 115, 5908-5917. (38) Safarik, D. J.; Meyer, R. J.; Mullins, C. B. Thickness dependent crystallization kinetics of sub-micron amorphous solid water films. $J$. Chem. Phys. 2003, 118, 4660-4671.

(39) Cheng, Y.; Grigorieff, N.; Penczek, P. A.; Walz, T. A primer to single-particle cryo-electron microscopy. Cell 2015, 161, 438-449.

(40) Karuppasamy, M.; Karimi Nejadasl, F.; Vulovic, M.; Koster, A. J.; Ravelli, R. B. G. Radiation damage in single-particle cryo-electron microscopy: effects of dose and dose rate. J. Synchrotron Radiat. 2011, $18,398-412$.

(41) Mariedahl, D.; Perakis, F.; Späh, A.; Pathak, H.; Kim, K. H.; Benmore, C.; Nilsson, A.; Amann-Winkel, K. X-ray studies of the transformation from high- to low-density amorphous water. Philos. Trans. R. Soc., A 2019, 377, 20180164.

(42) Russo, C. J.; Passmore, L. A. Ultrastable gold substrates for electron cryomicroscopy. Science 2014, 346, 1377-1380.

(43) Egerton, R. F.; Li, P.; Malac, M. Radiation damage in the TEM and SEM. Micron 2004, 35, 399-409.

(44) Andersson, O. Thermal conductivity of normal and deuterated water, crystalline ice, and amorphous ices. J. Chem. Phys. 2018, 149, 124506.

(45) Kouchi, A.; Greenberg, J. M.; Yamamoto, T.; Mukai, T. Extremely Low Thermal Conductivity of Amorphous Ice: Relevance to Comet Evolution. Astrophys. J. Lett. 1992, 388, L73. 